\title{
Evidence of noncollisional femtosecond laser energy deposition in dielectric materials
}

\author{
G. Duchateau $\odot,{ }^{*}$ B. Chimier, S. Coudert $\odot$, E. Smetanina, L. Barilleau, N. Fedorov, H. Jouin, G. Geoffroy, P. Martin, \\ and V. T. Tikhonchuk \\ Université de Bordeaux-CNRS-CEA, Centre Lasers Intenses et Applications, UMR 5107, 351 Cours de la Libération, 33405 Talence, France
}

(Received 9 July 2019; revised 18 June 2020; accepted 25 June 2020; published 9 July 2020)

\begin{abstract}
Electron dynamics in the bulk of large band gap dielectric crystals induced by intense femtosecond laser pulses at $800 \mathrm{~nm}$ is studied. With laser intensities (a few $10 \mathrm{TW} / \mathrm{cm}^{2}$ ) under the ablation threshold, electrons with unexpected energies in excess of $40-50 \mathrm{eV}$ are observed by using the photoemission spectroscopy. A theoretical approach based on the Boltzmann kinetic equation including state-of-the-art modeling for various particles interactions is developed to interpret these experimental observations. A direct comparison shows that both electron heating in the bulk and a further laser field acceleration after ejection from the material contribute equivalently to the final electron energy gain. The laser energy deposition in the material is shown to be significantly driven by a noncollisional process, i.e., direct multiphoton transitions between subbands of the conduction band. This work also sheds light on the contribution of the standard electron excitation/relaxation collisional processes, providing a new baseline to study the electron dynamics in dielectric materials and associated applications as laser material micromachining. To support such applications, a simple expression to evaluate the energy deposition by noncollisional absorption is provided.
\end{abstract}

DOI: 10.1103/PhysRevB.102.024305

\section{INTRODUCTION}

Developments of laser facilities delivering ultrashort and intense laser pulses with photon energy in the $\mathrm{eV}$ range have motivated studies in laser-solid interactions including metals [1,2], semiconductors [3], and dielectrics [4,5]. Focusing of a femtosecond laser pulse in a transparent dielectric material may induce modifications beneath the surface, which can be tailored to produce permanent three-dimensional localized structural changes [6-10]. Micromachining efficiency depends on the amount of laser energy deposited in the irradiated volume. A control of the amount and spatial shape of the deposited laser energy opens the way to a large variety of applications going from photonics, bulk microelectronics, nanofluidics, to medicine [11]. Together with advanced experimental setups, such a control can be achieved by an in-depth modeling description of the physical processes at play, i.e., laser driven electron dynamics leading to the energy deposition into the material. An accurate prediction of the laser energy deposition may further support the development of these applications and improve the knowledge of the fundamental laws governing the laser-solid interaction. So there is a strong need to accurately describe the electron dynamics in dielectric materials irradiated by femtosecond laser pulses with intensities ranging from a few $\mathrm{TW} / \mathrm{cm}^{2}$ to the ablation threshold.

The admitted picture for the laser energy deposition into the dielectric material is as follows. The laser energy is first absorbed by electrons through the processes of both ionization and excitation/relaxation in the conduction band. During the second stage, the absorbed laser energy is redistributed

\footnotetext{
*guillaume.duchateau@u-bordeaux.fr
}

between the excited carriers which may reach higher energies while they undergo collisions with phonons, ions, and other electrons in the presence of the laser field. These processes eventually lead to the energy transfer to the lattice. This is a collisional picture theoretically described either by the Drude model, multiple rate equations [12], or the kinetic Boltzmann equation $[4,5,13]$ which can provide the energy distribution of excited electrons related to the laser energy deposition. This distribution can be experimentally obtained through photoemission spectroscopy [14-17]. For laser intensities below the breakdown threshold of various dielectric materials including CsI, diamond, $\mathrm{CeF}_{3}$, sapphire, and $\mathrm{SiO}_{2}$, photoemitted electrons exhibit universal characteristics of energies in excess of tens of $\mathrm{eV}$ which collisional heating is not able to account for $[17,18]$. Following studies on high-order harmonics generation (HHG) in solids where such electron energies are reached [19-23], it has been suggested that the previous photoemission observations may be explained by direct multiphoton transitions between subbands of the conduction band, hereafter referred to as the interband process $[15,16]$. A question then arises on the importance of the interband process relative to collision-assisted electron transitions, and on its contribution to the laser energy deposition in dielectric materials (which is related to the electron energy distribution).

More generally, when a dielectric material is irradiated by a femtosecond laser pulse, two main classes of processes related to the electron dynamics are generally considered independently: material modifications induced by laser energy deposition [7] and HHG [19,20], corresponding to irreversible and reversible electron dynamics, respectively. In both cases, valence electrons are first promoted to excited states of the conduction band, the subsequent dynamics then departing between these two processes. HHG is a noncollisional process related to direct electron intra- and interband transitions. 
The coherence between the excited electrons with the laser electric field stands during at least the period of time of one optical cycle, eventually leading to the recombination of the excited electron to its parent ion (resulting in radiation emission). During the electron excursion in the conduction band, electrons may reach energies of tens of eV but only in a short period of time (this is a reversible process of energy exchange between electrons and fields). Another quantum pathway corresponds to the excitation of valence electrons to the bottom of the conduction band where they may undergo collisions (scattering with momentum exchange) with phonons or other electrons [24], then breaking their coherence with the laser electric field. These scattered electrons then may further undergo collisions, eventually leading to the laser energy deposition into the material (phonon density is increased through collisions). The relative magnitude of these two important processes depends on laser and material parameters.

Two main classes of models are candidate to address this question on the importance of the interband process relative to collision-assisted electron transitions leading to the laser energy deposition in dielectric materials. (i) Collisional models as solving the state-of-the-art quantum Boltzmann equation, including all possible electronic excitation and relaxation processes, provide the electron energy distribution [4,5,12]. But the interband process has never been included except in [13]. (ii) Noncollisional models based on a resolution of the time-dependent Schrödinger equation or optical Bloch equations $[20-23,25]$. The former allows one to interpret the photoemission observation but the collisions are not included $[15,16]$. Despite these studies, a direct comparison between theoretical and experimental electron energy distributions has never been performed while this is an indispensable step for validating any model $[4,5,12,15]$, leaving serious interrogations regarding the (nonequilibrium) electron dynamics in the conduction band of dielectric materials.

In the present work the electron dynamics is studied both experimentally and theoretically for $\alpha$-quartz (crystalline $\mathrm{SiO}_{2}$ ) which is a representative example of large band gap dielectrics. The experimental setup and the observed photoelectron energy distributions for various laser intensities are presented in Sec. II. The theoretical model providing a kinetic description of the laser induced electron dynamics both inside the bulk and after ejection is provided in Sec. III. It is based on a resolution of the quantum Boltzmann equation including all possible bulk excitation/relaxation processes coupled to a subsequent laser driven field acceleration (LDFA) of electrons after their ejection [26]. In Sec. IV the observed photoelectron energy distributions are directly compared with the theoretical predictions, within the challenging accuracydemanding linear scale in the present context. We emphasize that only the maximum energy of ejected electrons has been considered in previous works $[13,17,18]$. The present model allows us to predict photoelectron spectra which are in a good agreement with experimental results for various laser intensities. The noncollisional direct multiphoton transitions between subbands of the conduction band make a significant contribution of heating of electrons in dielectric materials in contrast to the widely used assumption of a dominant role of collisional processes including phonon-assisted photon

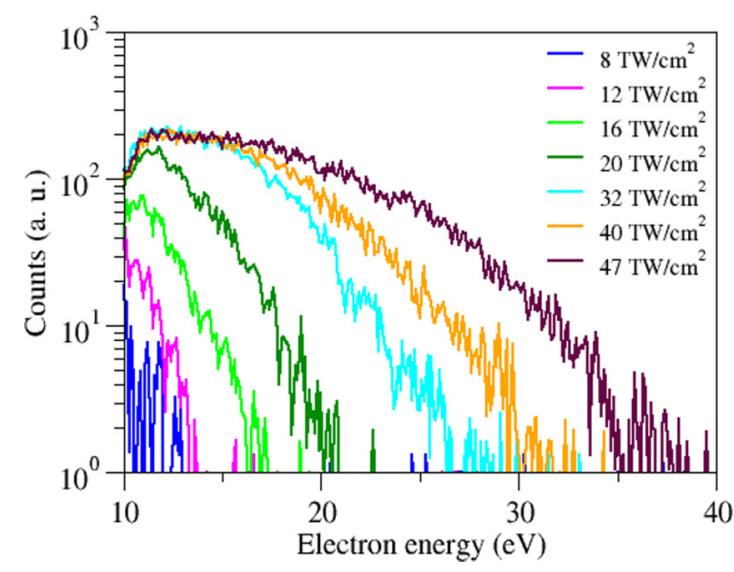

FIG. 1. Experimental photoelectron energy distribution for quartz. Only the region of highest electron energies is shown, where dynamics is only due to intrinsic processes modeled in the present study (see text for more details).

absorption and inverse Bremsstrahlung. Our model accounts in particular for acceleration of electrons to energies in excess of $40 \mathrm{eV}$ for laser intensities below the ablation threshold. For application purpose as laser micromachining of materials, a simple expression to evaluate the energy deposition by noncollisional absorption is provided. Finally, conclusions of this work are drawn in Sec. V.

\section{EXPERIMENTAL OBSERVATIONS}

The experiment is carried out on the CELIA Aurore Ti:saphire laser facility [27]. A $1 \mathrm{~mm}$ thick $\alpha$-quartz target is irradiated by linearly $P$-polarized pulses at the wavelength of $\lambda=800 \mathrm{~nm}$, with $70 \mathrm{fs}$ duration (full width at half maximum, FWHM) at $1 \mathrm{kHz}$ repetition rate. The incident angle is $45^{\circ}$ and a $30 \mathrm{~mm}$ lens produced a Gaussian intensity distribution in a $21 \mu \mathrm{m}$ spot size (FWHM). The experiment is conducted in a vacuum chamber at a pressure of $10^{-9}$ Torr, and the sample is heated homogeneously to a temperature of $800 \mathrm{~K}$ to maximize the photoemission yield, by decreasing the surface charge. The photoelectrons emitted from the surface are collected by a hemispherical analyzer (CLAM IV VG Microtech) with 9 channeltrons operating in ultraviolet photoelectron spectroscopy mode. The axis of the detector is perpendicular to the sample surface.

Figure 1 shows the experimental photoemission spectra for quartz obtained with intensities ranging from 8 to $47 \mathrm{TW} / \mathrm{cm}^{2}$. In general, such spectra exhibit a main peak, for an electron energy of a few eV, which almost does not evolve with respect to the intensity. This peak corresponds to secondary electrons in which properties depend on the surface state. Since we are interested in intrinsic processes corresponding to high enough energies, the low energy region is not shown. Above roughly $11 \mathrm{eV}$, the signal exhibits a smooth decrease up to a maximal energy $E_{\max }$ for which at least one count is measured. $E_{\max }$ increases with respect to the intensity and reaches roughly $40 \mathrm{eV}$ for the highest intensity. Note that after each photoemission experiment we have checked by using a microscope that there was no modification of the target surface, as observed in [28]. Similar behaviors in terms 
of high energies and distribution shape have been obtained for other large band gap materials as sapphire, CsI, diamond, and $\mathrm{CeF}_{3}[17,18]$.

\section{KINETIC MODELING OF THE LASER INDUCED ELECTRON DYNAMICS}

The electron dynamics in the bulk is described by a Boltzmann kinetic equation which domain of validity is fulfilled by using moderate laser intensities producing electron densities of the order of $10^{18}-10^{20} \mathrm{~cm}^{-3}[5,12,13]$. This particle density is small enough to ensure only binary collisions and an average distance between particles larger than the De Broglie length allowing one to consider particles as evolving classically (Boltzmann equation is classical, whereas collision operators are evaluated through quantum calculations). On the other hand, such densities are large enough to ensure a sufficiently large number of collisions. Depending on the collisional process, the collision frequency is in the range between $10^{14}$ and $10^{15} \mathrm{~s}^{-1}$. Since the laser pulse duration is $70 \mathrm{fs}$, at least several collisions on average for each process take place during the interaction. The use of a distribution function thus makes sense and each collisional process is expected to have a particular influence on the shape of the distribution function.

Electrons are ejected from a nanometer-size layer beneath the target surface where the laser electric field can be considered as a constant. Indeed, the field amplitude adapts to the dielectric material property on a length scale $v_{b} / \omega_{v e}$ where $v_{b}$ is the velocity of bound valence electrons and $\omega_{v e}$ is their plasma frequency. The order of magnitude of these quantities is $3 \times 10^{6} \mathrm{~m} \mathrm{~s}^{-1}$ and $10^{16} \mathrm{~s}^{-1}$, respectively, leading to $v_{b} / \omega_{v e} \simeq 3 \AA$. The laser intensity is thus relatively constant a few nanometers beneath the surface, implying no spatial dependence in the electron distribution $f(\vec{k}, t)$, where $\vec{k}$ is the momentum and the electron energy is $E_{k}=\hbar^{2} \vec{k}^{2} / 2 m_{e}$. The temporal evolution of $f$ in the bulk is then given by

$$
\frac{\partial}{\partial t} f(\vec{k}, t)=\left.\frac{\partial f(\vec{k}, t)}{\partial t}\right|_{\text {ioniz }}+\left.\frac{\partial f(\vec{k}, t)}{\partial t}\right|_{\text {relax }}+\left.\frac{\partial f(\vec{k}, t)}{\partial t}\right|_{\text {heat }}
$$

where the three collision integrals on the right-hand side describe the ionization, the relaxation, and the laser excitation of conduction electrons, respectively. The electron distribution is assumed to be isotropic since it is due to electron collisions with acoustic phonons in which characteristic timescale is 10 fs [29].

The ionization processes consist of both the photoionization, which is evaluated through the complete Keldysh expression [30], and the impact ionization as described in [4]. The relaxation processes are related to electron-electron (e-e) [4] and electron-phonon (e-ph) [4,29] collisions, which induce the energy exchange of electrons between themselves and the lattice, respectively. These collision integrals are calculated with Fermi's golden rule. The electron recombination is also included with a characteristic time of $150 \mathrm{fs}$ [31] which is assumed not to depend on $E_{k}$. The energy distribution of phonons is assumed not to evolve during this short interaction

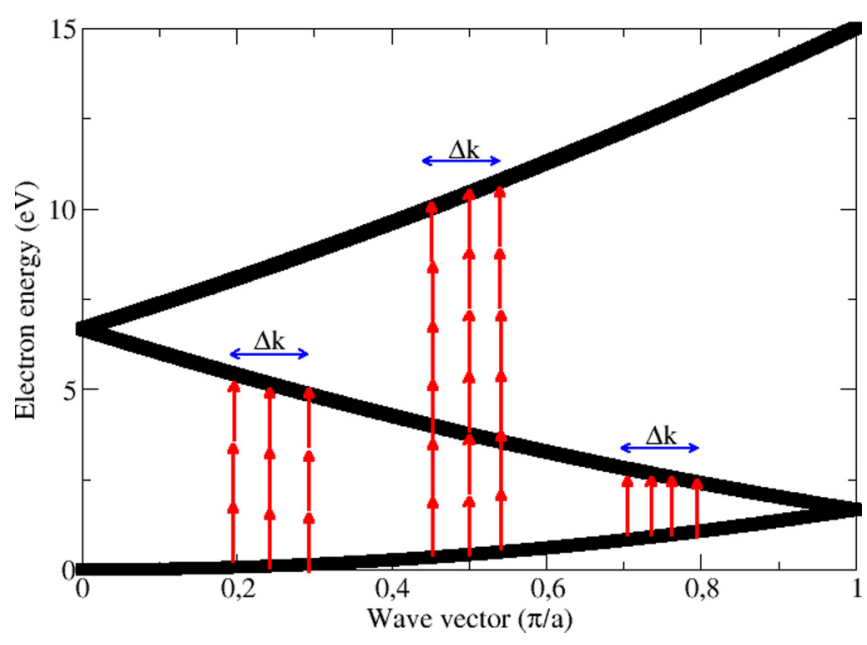

FIG. 2. Illustration of the structure of the conduction band in the first Brillouin zone as described in the multiple parabolic band model. The bands exhibit an energy bandwidth of $h v_{c}$ of the order of $1 \mathrm{eV}$ due to the collisional broadening. An illustration of possible multiphoton transitions is depicted by the red arrows. Due to the broadening, the transitions take place over a wave vector region $\Delta k$.

time and is set to the equilibrium Bose-Einstein distribution with a lattice temperature set to $800 \mathrm{~K}$.

Two main processes are included for the laser-induced excitation of conduction electrons. First, the electrons can absorb or emit simultaneously several photons during a collision with phonons (e-ph-pt) or ions (e-ion-pt, inverse Bremsstrahlung) [4]. Second, electron excitation can also take place through a noncollisional process (no other particle as ion or phonon is involved to absorb photons) which is direct multiphoton interband transitions [13,15,17,18,32]. To include the latter process, the conduction band is described by multiple parabolic energy subbands. An illustration of this process is provided by Fig. 2. Note this mechanism departs from the Rethfeld's approach $[4,12]$ and is expected to have a significant impact on the electron dynamics. The interband rate is evaluated according to the expression and parameters provided in [13], i.e., without using any fitting procedure. Such an approach allows us to introduce explicitly the collisionless heating in a full kinetic treatment of the electron dynamics in laser-driven dielectrics [13].

Note that the photoionization process and the laser induced electron dynamics in the conduction band are described separately within the present approach, whereas it may be treated on the same foot by solving optical Bloch equations for instance [20-23,25]. Both processes are introduced separately because (i) they are of different nature and (ii) they cannot be modeled within the Boltzmann framework on the same foot. (i) They are of different nature in the sense that the multiphoton ionization process bridges a bound valence state to a quasifree continuum state, whereas the multiphoton excitation in the conduction band bridges two continuum states. Despite the starting point of the theory to evaluate these collision rates is similar, the dipolar matrix elements are different, justifying to introduce both terms in the Boltzmann equation. (ii) The photoionization process generates electrons in the conduction band, whereas the multiphoton excitation process 
in the conduction band leaves the total conduction electron density unchanged. The latter process only changes the electron energy distribution in the conduction band. Within our Boltzmann model, only one parabolic conduction band is considered so that the photoionization process corresponds to a source term of electrons into the bottom of the conduction band. An additional Boltzmann equation for the valence band may be added for the sake of generality and treating on the same foot both multiphoton processes, but its influence is expected to be small since no more than a few percent of valence electrons are promoted to the conduction band. This approach is explained in detail in [13].

Solution to the Boltzmann equation (1) provides the energy distribution of electrons in the material. Their ejection from the surface is possible if their energy is larger than the work function which is $0.9 \mathrm{eV}$ for quartz [33,34]. For low energy ejection, surface effects may modify the distribution [35]. However, this influence is negligible for the most energetic electrons which are considered here. Consequently, the distribution of ejected electrons near the surface is assumed to be the same as the one calculated in the bulk. To obtain a distribution directly comparable to the experimental data, $f\left(E_{k}, t\right)$ is first weighted by the density of states $g\left(E_{k}\right) \propto \sqrt{E_{k}}$ accounting for a three-dimensional free electron gas. Second, the influence of the laser electric field $F(t)$, which may further accelerate or decelerate the ejected electrons depending on their instant of emission, is taken into account: it is a laserdriven field acceleration (LDFA) which can change the energy distribution [26]. The final energy of the ejected electron is obtained by integrating the classical equation of the electron motion in vacuum $d v / d t=-e F(t) / m_{e}$ from the ejection moment $t_{e}$ to the end of the laser pulse (160 fs in practice). The initial ejection velocity $v_{0}$ at $t_{e}$ is evaluated from the calculated electron distribution in the bulk. Since electrons in the bulk undergo numerous collisions before ejection, they lose any coherence [24] with the laser electric field at the time of ejection. Consequently, the electrons are assumed to be ejected uniformly during the interaction: their ejection time is not related to any particular phase of the laser electric field. Since in experiments ejected electrons are collected over the whole laser pulse duration, the theoretical predictions are obtained by integrating the electron distribution over time. Note that the maximum energy gain corresponds to the classical energy of half an optical cycle. For an electron ejected at the optimal time, a simple calculation shows that the final energy is roughly $40 \mathrm{eV}$ for an ejection energy $E_{k_{0}}=20 \mathrm{eV}$ and a laser intensity $I=50 \mathrm{TW} / \mathrm{cm}^{2}$.

\section{EVIDENCE OF NONCOLLISIONAL LASER ENERGY DEPOSITION}

Figure 3 shows, within a linear scale, the experimental distributions of ejected electrons together with the theoretical predictions for intensities ranging from 8 to $47 \mathrm{TW} / \mathrm{cm}^{2}$. The theoretical distribution at $I=24 \mathrm{TW} / \mathrm{cm}^{2}$ is multiplied by a renormalization factor to compare to the experimental data. The same factor is used for all intensities. Both experimental and theoretical data are in a good agreement for each intensity. The only significant discrepancy between modeling and experimental spectra appears for electron energies below $17 \mathrm{eV}$

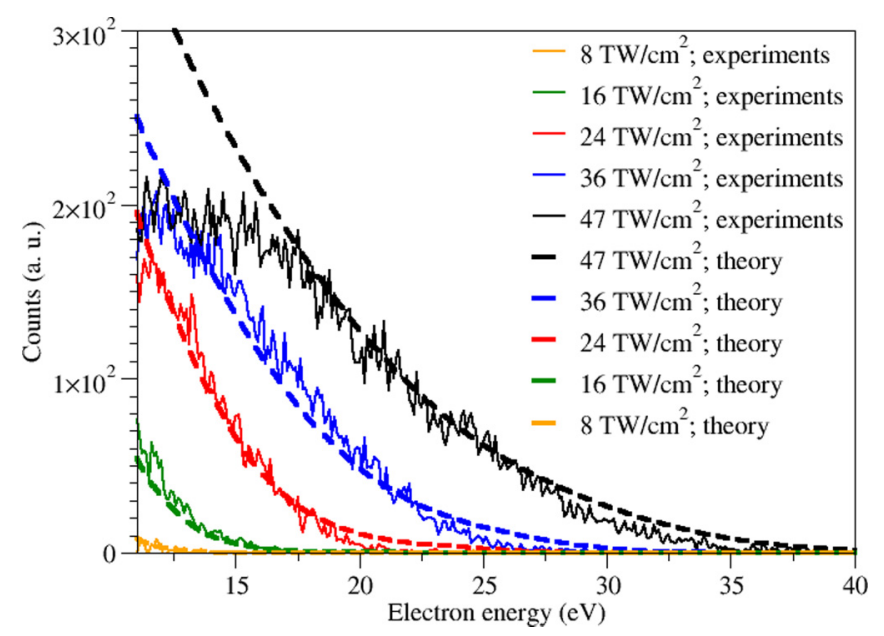

FIG. 3. Energy distributions of ejected electrons from photoemission experiments (solid lines) and modeling (dashed lines) for various laser intensities.

for the largest laser intensity. It may be attributed to electron transport in the bulk which is not included in the present modeling: low energy electrons are sensitive to spatial charge rearrangements (potential minimization) in the bulk which are all the more important that the produced charge density is large (or highest laser intensities).

The experimental observations can be reproduced only if we include all above-mentioned physical processes: photoionization, impact ionization, heating through electron-phononphoton and interband transitions, the relaxation through electron-phonon and electron-electron collisions, and the LDFA. In order to evaluate the role of each process on the electron dynamics in the bulk, they have been successively switched off. The comparison of the theoretical spectra obtained with the various modeling configurations (not shown here) to the experimental data leads to the following conclusions. (i) the impact ionization prevents electrons from reaching too high energies at the largest intensities. However, there is no electron avalanche, which is consistent with the fact that the irradiated material is not damaged. (ii) Regarding the electron heating in the conduction band, the introduction of both e-ph-pt and interband processes is required to recover correct slopes for all considered intensities. In particular, the interband process enables us to mimic the smooth decrease with respect to the electron energy [13]. (iii) Regarding the relaxation, the electron-electron collisions provide a smooth energy distribution. Otherwise, the electron distribution contains several peaks separated by the photon energy [13] that is not experimentally observed. The contribution of e-ph collisions also permits us to redistribute electrons to lower energies providing the observed slopes. In contrast, the electron recombination and e-ion-pt do not modify significantly the spectra due to the short interaction time and relatively low ionization degree (the electron density in the conduction band is in between $10^{19}$ and $10^{20} \mathrm{~cm}^{-3}$ depending on the intensity), respectively.

Figure 4 provides the evolution of $E_{\max }$ as a function of the laser intensity from experimental observations and as predicted by the modeling (the interband and LDFA processes 


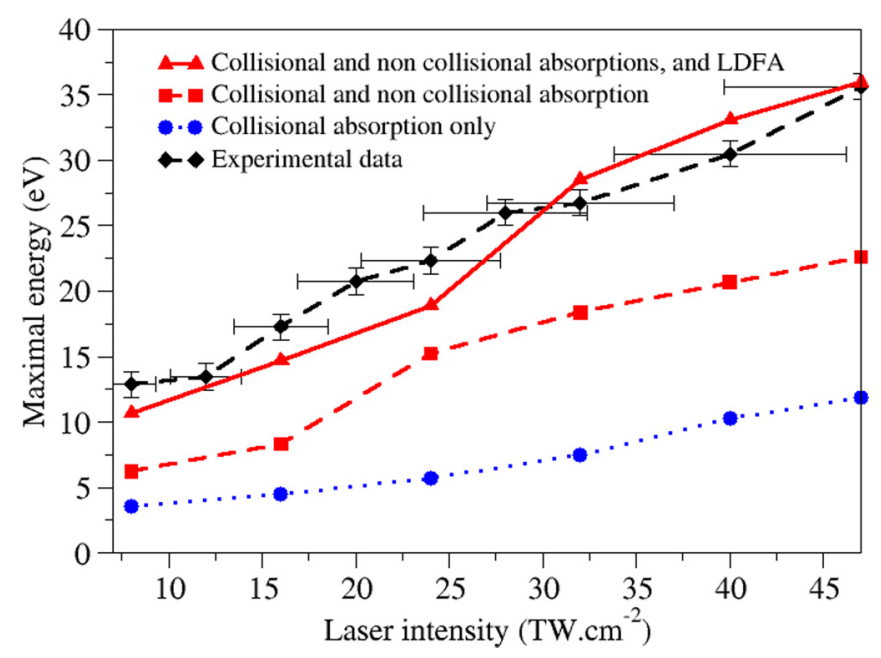

FIG. 4. Maximum energy of photoemitted electrons as a function of the laser intensity. Absorption processes are gradually switched on within the modeling, see inset legend for curves meaning.

are included or not). In the experiment, $E_{\max }$ increases monotonically from 11 to roughly $40 \mathrm{eV}$. Without the interband and LDFA processes, $E_{\max }$ cannot exceed $10 \mathrm{eV}$ for the highest intensity, the heating being only due to e-ph-pt collisions in that case. By including the interband process, $E_{\max }$ reaches about $23 \mathrm{eV}$, i.e., twice the energy of the previous configuration. Both e-ph-pt and interband contributions to the electron heating are thus comparable. A good agreement with the experimental data is obtained when the LDFA is included, providing an enhancement of the final electron energy of more than a factor of 2 . These considerations clearly show that the observed photoemission spectra result from three physical processes with comparable contributions. All the previous conclusions are expected to be similar for the abovementioned other large band gap dielectric materials which exhibit similar band structures and coupling between states.

We have shown the importance of the noncollisional laser heating. For applicative purposes as laser micromachining of materials, a simple expression to evaluate the laser energy deposition is desirable. The starting point is the interband rate for an electron $w_{1 f}(n)$ bridging the bottom subband 1 and the final subband $f$, as derived in [13]

$$
w_{1 f}=\frac{m}{4 \pi \hbar^{2}\left|p_{f}\right|} V_{1 f}^{2} I J_{n}^{\prime 2}\left(B_{1 f}\right)\left[\left(\frac{\pi}{a}\right)^{2}-k_{n}^{2}\right],
$$

where $n$ is the number of photons required to bridge resonantly subbands 1 to $f$ at wave vector $k_{n}$. $J_{n}^{\prime}$ is the derivative of the Bessel function with argument $B_{1 f}=\frac{1}{\hbar \omega} \frac{e \vec{F}(t)\left(\vec{p}_{f}-\vec{p}_{1}\right)}{m \omega}$, $\vec{p}_{i}$ being momenta related to the reciprocal-lattice vector. $V_{1 f}$ is the dipolar matrix element. More details on notations and values of parameters relative to this interband rate can be found in [13].

The energy rate is obtained by multiplying Eq. (2) by $\hbar \omega$ and summing over all allowed multiphoton orders. It then has to be weighted by $\Delta k /(\pi / a)=2 m a^{2} h v_{c} / \pi^{2} \hbar^{2}$ that is the relative part of the Brillouin zone participating to interband transition due to the collisional broadening (see Fig. 2). Finally, the energy density absorbed per unit of time through the

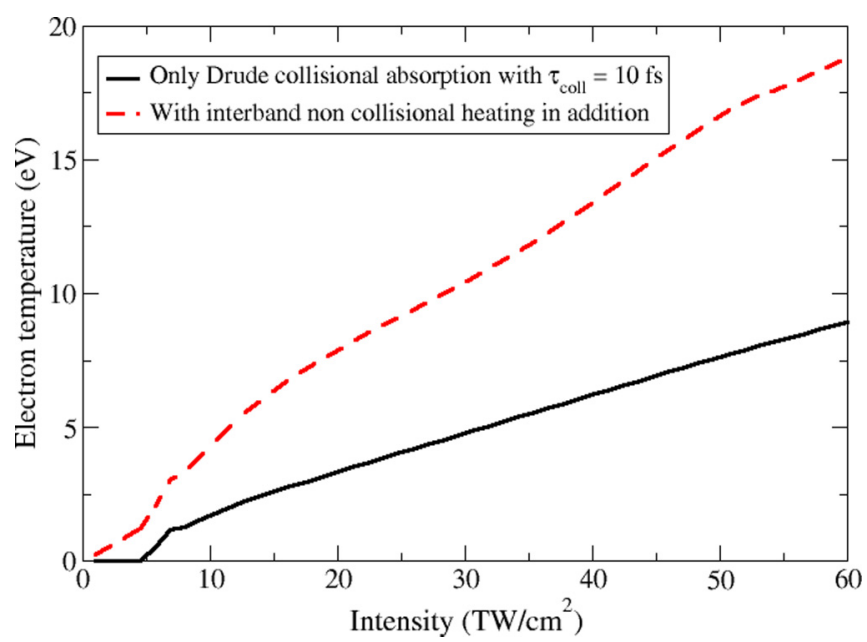

FIG. 5. Evolution of the electron temperature as a function of the maximum laser intensity as estimated with a simplified Drude-like modeling.

interband process $d U_{n c} / d t$ reads

$$
\frac{d U_{n c}}{d t}=\frac{m a \omega n_{e}(t) \Delta k}{4 \pi^{2} \hbar\left|p_{f}\right|} V_{1 f}^{2} I \sum_{n} n J_{n}^{\prime 2}\left(B_{1 f}\right)\left[\left(\frac{\pi}{a}\right)^{2}-k_{n}^{2}\right],
$$

where $n_{e}(t)$ is the electron density in the conduction band. In order to check the reliability of the expression (3) against kinetic calculations and compare its contribution relative to standard collisional absorption, an evaluation of the electron temperature is performed. The conduction electron density is evaluated by solving multiple rate equations as presented in [12]. By setting the Drude averaged collision time to $v_{c}^{-1}=$ $10 \mathrm{fs}$ accounting mainly for electron-phonon collisions, the evolutions of the electron temperature $\left(=U / C_{e}\right.$ with $C_{e}$ the classical heat capacity) as a function of the intensity including or not the noncollisional laser heating are obtained. Figure 5 shows these results at the end of the interaction where the electron distribution has sufficiently relaxed to an equilibrium state allowing one to define the temperature. They exhibit similar trends as those provided by solving the quantum Boltzmann equation [13], and values consistent with the present electron energies (because $k_{B} T_{e} \simeq E_{\max } / 2$ within the present conditions [13]). This demonstrates the reliability of this simplified model [Eq. (3)] describing the additional contribution of noncollisional heating to the Drude description.

\section{CONCLUSION}

Photoemission experiments have been carried out with large band gap dielectric crystals irradiated by near infrared laser femtosecond pulses with intensities below the ablation threshold. The electron energy spectra exhibit a long tail up to energies close to $40 \mathrm{eV}$ for the highest intensities. The underlying electron dynamics has been analyzed through a state-of-the-art modeling based on the Boltzmann kinetic equation including the main excitation/relaxation processes, and the laser driven field acceleration of ejected electrons. Experimental and theoretical energy distributions are in a very good agreement for all considered laser intensities, whereas up to now only the maximum energy of ejected electrons 
was considered. This direct comparison of the theoretical predictions to the whole experimental electron energy distributions shows that both heating in the bulk and electric field acceleration in the vacuum make comparable contributions to the electron energy gain. The noncollisional direct multiphoton transitions between subbands of the conduction band is a major mechanism for electron heating in the bulk of dielectric materials which must be included for the evaluation of the energy deposition. For application purpose as laser micromachining of materials, a simple expression to evaluate the energy deposition by noncollisional absorption has been provided.

\section{ACKNOWLEDGMENTS}

This project has received funding from the European Union's Horizon 2020 research and innovation programme under Grant Agreement No. 654148 Laserlab-Europe. Henri Bachau is acknowledged for fruitful discussions on high-order harmonics generation in solids.
[1] B. Rethfeld, A. Kaiser, M. Vicanek, and G. Simon, Phys. Rev. B 65, 214303 (2002).

[2] B. Scharf, V. Perebeinos, J. Fabian, and P. Avouris, Phys. Rev. B 87, 035414 (2013).

[3] P. E. Selbmann, M. Gulia, F. Rossi, E. Molinari, and P. Lugli, Phys. Rev. B 54, 4660 (1996).

[4] A. Kaiser, B. Rethfeld, M. Vicanek, and G. Simon, Phys. Rev. B 61, 11437 (2000).

[5] N. S. Shcheblanov and T. E. Itina, Appl. Phys. A 110, 579 (2013).

[6] E. N. Glezer, M. Milosavljevic, L. Huang, R. J. Finlay, T.-H. Her, J. P. Callan, and E. Mazur, Opt. Lett. 21, 2023 (1996).

[7] E. G. Gamaly, Phys. Rep. 508, 91 (2011).

[8] A. Marcinkevičius, S. Juodkazis, M. Watanabe, M. Miwa, S. Matsuo, H. Misawa, and J. Nishii, Opt. Lett. 26, 277 (2001).

[9] R. R. Gattass and E. Mazur, Nat. Photonics 2, 219 (2008).

[10] J. Zhang, M. Gecevicius, M. Beresna, and P. G. Kazansky, Phys. Rev. Lett. 112, 033901 (2014).

[11] R. Menzel, Photonics: Linear and Nonlinear Interactions of Laser Light and Matter (Springer, Berlin, 2001).

[12] B. Rethfeld, Phys. Rev. Lett. 92, 187401 (2004).

[13] L. Barilleau, G. Duchateau, B. Chimier, G. Geoffroy, and V. Tikhonchuk, J. Phys. D: Appl. Phys. 49, 485103 (2016).

[14] P. Dombi, A. Horland, P. Racz, I. Marton, A. Trugler, J. R. Krenn, and U. Hohenester, Nano Lett. 13, 674 (2013).

[15] B. N. Yatsenko, H. Bachau, A. Belsky, J. Gaudin, G. Geoffroy, S. Guizard, P. Martin, G. Petite, A. Philippov, and A. N. Vasil'ev, Phys. Status Solidi C 2, 240 (2005).

[16] H. Bachau, A. N. Belsky, I. B. Bogatyrev, J. Gaudin, G. Geoffroy, S. Guizard, P. Martin, Yu. V. Popov, A. N. Vasil'ev, and B. N. Yatsenko, Appl. Phys. A 98, 679 (2009).

[17] A. Belsky, H. Bachau, J. Gaudin, G. Geoffroy, S. Guizard, P. Martin, G. Petite, A. Philippov, A. N. Vasil'ev, and B. N. Yatsenko, Appl. Phys. B 78, 989 (2004).
[18] A. Belsky, P. Martin, H. Bachau, A. N. Vasil'ev, B. N. Yatsenko, S. Guizard, G. Geoffroy, and G. Petite, Europhys. Lett. 67, 301 (2004).

[19] G. Vampa, C. R. McDonald, G. Orlando, D. D. Klug, P. B. Corkum, and T. Brabec, Phys. Rev. Lett. 113, 073901 (2014).

[20] G. Ndabashimiye, S. Ghimire, M. Wu, D. A. Browne, K. J. Schafer, M. B. Gaarde, and D. A. Reis, Nature (London) 534, 520 (2016).

[21] D. Golde, T. Meier, and S. W. Koch, Phys. Rev. B 77, 075330 (2008).

[22] M. Wu, S. Ghimire, D. A. Reis, K. J. Schafer, and M. B. Gaarde, Phys. Rev. A 91, 043839 (2015).

[23] P. G. Hawkins, M. Yu. Ivanov, and V. S. Yakovlev, Phys. Rev. A 91, 013405 (2015).

[24] A. Bourgeade and G. Duchateau, Phys. Rev. E 85, 056403 (2012).

[25] J. B. Khurgin, J. Opt. Soc. Am. B 33, C1 (2016).

[26] P. Mulser, S. M. Weng, and T. Liseykina, Phys. Plasmas 19, 043301 (2012).

[27] V. Bagnoud and F. Salin, Appl. Phys. B 70, S165 (2000).

[28] D. Du, X. Liu, G. Korn, J. Squier, and G. Mourou, Appl. Phys. Lett. 64, 3071 (1994).

[29] Ph. Daguzan, P. Martin, S. Guizard, and G. Petite, Phys. Rev. B 52, 17099 (1995).

[30] L. V. Keldysh, Zh. Eksp. Teor. Fiz. 47, 1945 (1964) [Sov. Phys. JETP 20, 1307 (1965)].

[31] P. Martin, S. Guizard, P. Daguzan, G. Petite, P. D'Oliveira, P. Meynadier, and M. Perdrix, Phys. Rev. B 55, 5799 (1997).

[32] A. Belsky, A. N. Vasil'ev, B. N. Yatsenko, H. Bachau, P. Martin, G. Geoffroy, and S. Guizard, J. Phys. IV France 108, 113 (2003).

[33] V. S. Kortov and S. V. Zvonarev, J. Surf. Investigation 5, 764 (2011).

[34] K. Ohya, K. Inai, H. Kuwada, T. Hayashi, and M. Saito, Surf. Coat. Technol. 202, 5310 (2008).

[35] C. Mariani, Conf. Proc. Italian Phys. Soc. 82, 211 (2003). 